sometimes unaware of the difficulties that general practitioners may face in obtaining the information required.

Marinker et al have recommended the development of protocols for referral between general practitioners and consultants. ${ }^{5}$ Some departments of child psychiatry now use standardised referral forms with specific questions on the attitude of the family to referral and involvement of other agencies. This study has shown that child psychiatrists have some special requirements for referral letters, but they are unlikely to obtain the desired information unless they educate general practitioners.
I am indebted to Maurice Place for his support and advice at every stage of this project.

1 Williams P, Wallace BB. General practitioners and psychiatrists-do they communicate? BMf 1974;i:505-7.

2 Pullen IM, Yellowlees AJ. Is communication improving between general practitioners and psychiatrists? $B M \mathcal{F} 1985 ; 290: 31-3$.

3 Kentish R, Jenkins P, Lask B. Study of written communication between general practitioners and departments of child psychiatry. $\exists R$ Coll Gen Pract 1987;37:162-3

4 Newton J, Eccles M, Hutchinson A. Communication between general practitioners and consultants: what should their letter contain? $B M 7$ 1992;304: $821-4$

5 Marinker $M$, Wilkin D, Metcalfe DH. Referral to hospital: can we do better? $B M F$ 1988;297:461-4.

(Accepted 21 fanuary 1993)

\title{
Drug misusers in Lothian: changes in injecting habits 1988-90
}

\section{Shay Griffin, Andy Peters, Margaret Reid}

Community Drug Problem Service, Royal Edinburgh Hospital, Edinburgh EH10 5BT

Shay Griffin, clinical assistant Andy Peters, chartered clinical psychologist Margaret Reid, research psychologist

\section{Correspondence to:}

Dr Griffin.

BMF 1993;306:693
Of $941 \mathrm{HIV}$ seropositive cases reported in Edinburgh up to September 1992, $515(55 \%)$ were associated with injecting drug misuse. ${ }^{1} \mathrm{HIV}$ and drug targeted services in the region have concentrated on a "harm reduction" approach, one aspect of which is to encourage injectors at least to use a safe injecting technique but preferably to switch to oral use. The aim of this study was to verify a strong clinical impression that injecting drug misuse in Lothian has recently diminished.

\section{Subjects, methods, and results}

The community drug problem service, the setting for this study, has been described. ${ }^{2}$ The data presented were drawn from detailed clinical interview of new patients at the point of initial assessment. Fifty consecutive referrals in 1988 were compared with 50 consecutive referrals in 1990 with respect to demographic characteristics and patterns of drug misuse. These included injecting frequency (past and recent), prescription status at assessment, drugs reportedly used, and syringe sharing. Injecting frequency was defined as never (no injecting), occasional (averaging fewer than two injecting episodes a week), and frequent (averaging two or more episodes a week). "Recent" injecting refers to the six months before assessment. "Past" injecting refers to the injecting career before that period.

The two samples did not differ in sex, age, employment status, proportion with a regular partner, source of referral, age at first drug misuse (excluding cannabis, solvents, and alcohol), or age at first injecting (among injectors).

The table shows significant differences between the two years in past injecting frequency $\left(\chi^{2}=19 \cdot 9, \mathrm{df}=2\right.$;

Comparison of 1988 and 1990 samples for past injecting frequency (whole samples), recent injecting frequency among past injectors only, and frequency of recent injecting among frequent past injector

\begin{tabular}{|c|c|c|c|c|c|c|}
\hline \multirow[b]{3}{*}{ Injecting frequency ${ }^{\star}$} & \multirow{2}{*}{\multicolumn{2}{|c|}{ Past injectingt }} & \multicolumn{4}{|c|}{ Recent injecting $\ddagger$} \\
\hline & & & \multicolumn{2}{|c|}{$\begin{array}{l}\text { Past intravenous } \\
\text { drug misusers }\end{array}$} & \multicolumn{2}{|c|}{$\begin{array}{l}\text { Frequent past } \\
\text { intravenous } \\
\text { drug misusers }\end{array}$} \\
\hline & 1988 & 1990 & 1988 & 1990 & 1988 & 1990 \\
\hline No (\%) never & $2(4)$ & $13(27)$ & $6(13)$ & $17(50)$ & $6(13)$ & $12(48)$ \\
\hline No $(\%)$ occasional & $1(2)$ & $9(18)$ & $6(13)$ & $11(32)$ & $6(13)$ & $8(3)$ \\
\hline No $(\%)$ frequent & $47(94)$ & $27(55)$ & $36(75)$ & $6(18)$ & $35(74)$ & $5(20)$ \\
\hline $\mathrm{p}$ Value & \multicolumn{2}{|c|}{$<0.0001$} & \multicolumn{2}{|c|}{$<0.0001$} & \multicolumn{2}{|c|}{$<0.0001$} \\
\hline
\end{tabular}

* See text for definitions.

tRefers to injecting history more than six months before assessment.

$\ddagger$ Refers to injecting history in six months before assessment. $\mathrm{p}<0.0001)$, recent injecting frequency $\left(\chi^{2}=26 \cdot 6\right.$, $\mathrm{df}=2 ; \mathrm{p}<0.0001)$, and recent injecting frequency among frequent past injectors $\left(\chi^{2}=19 \cdot 9, \mathrm{df}=2\right.$; $p<0.0001)$. This last finding excludes the possibility that the preponderance of frequent past injectors in 1988 could account for differences between 1988 and 1990 in recent injecting frequency.

Among recent injectors $37 \%$ (13/35) had recently shared syringes in 1988 compared with $20 \%$ (3/15) in 1990. This difference was not significant. Among opioid misusers $51 \%(24 / 47)$ in 1988 and $70 \%(30 / 43)$ in 1990 were receiving prescribed opioids before assessment. Among benzodiazepine misusers 70\% (23/33) in 1988 and $59 \%(23 / 39)$ in 1990 were being prescribed benzodiazepines before assessment. These differences were not significant. (Some patients reported being prescribed both drug types.)

Of the drugs in use within one month before assessment, injectable opioids such as Temgesic (buprenorphine) were significantly more commonly reported in 1988 than in 1990. Dihydrocodeine (rather than methadone) was the non-injectable opioid most commonly reported in both years but was significantly more popular in 1990 than in 1988.

The 1988 and 1990 samples were combined to test for association between prescribing status before assessment and frequency of recent injecting. Receiving a benzodiazepine prescription was not associated with recent injecting frequency. However, being prescribed opioids before assessment was strongly associated with lower recent injecting frequency $\left(\chi^{2}=25 \cdot 7\right.$, $\mathrm{df}=2 ; \mathrm{p}<0.0001)$.

\section{Comment}

In this study sample sizes were small. Nevertheless, it is clear that something has happened in Lothian to diminish injecting prevalence among clinic attenders. Reported opioid misuse seems to reflect this trend. Being prescribed oral opioids before assessment was associated with less recent injecting but as levels of such prescribing were similar in the two samples this would not, as a "treatment effect," fully explain the differences found in recent injecting frequency. Availability of oral opioids on the "grey market" to drug users not receiving a prescription may be a factor. Other possible factors may include the influence of public and drug user targeted education and changes in attitude owing to drug users' personal experience of friends and relatives becoming ill and dying of HIV related conditions.

Study of an "out of treatment" group might disclose to what extent, if at all, the trends reported reflect behaviour change in the wider population of drug misusers in Lothian.

1 Communicable Diseases (Scotland) Unit, Ruchill Hospital, Glasgow. AIDS Weekly News Supplement 1992 Sep 30: No 272.

2 Greenwood J. Creating a new drug service in Edinburgh. BMF 1990;300:587-9.

(Accepted 7 fanuary 1993) 